\title{
The diagnostic and prognostic value of five blood markers in cutaneous T-cell lymphomas: a validation cohort
}

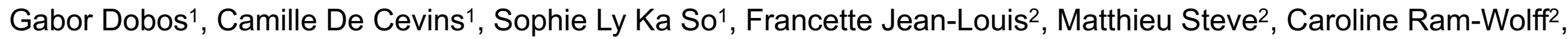 \\ Matthieu Resche-Rigon ${ }^{3}$, Armand Bensussan ${ }^{1}$, Martine Bagot ${ }^{2}$ and Laurence Michel ${ }^{1 *}$ and GELFC ${ }^{4}$ members
}

1INSERM U976 Human Immunology, Pathophysiology and Immunotherapy, Skin Research Institute, Hôpital Saint-Louis, Université de Paris, France ${ }^{2}$ Department of Dermatology, Hôpital Saint-Louis, APHP France

${ }^{3}$ Department of Biostatistics and Medical Information, SBIM, Hôpital Saint-Louis, APHP France 4 GFELC : Goupe Français d'Etude des Lymphomes Cutanés

\section{INTRODUCTION}

Clinical and laboratory diagnostics of mycosis fungoides (MF), the most common cutaneous lymphoma, is challenging. Our previous work described 4 promising blood markers of Sézary syndrome (SS): T-plastin, Twist, NKp46 and KIR3DL2 (Michel et al. Blood 2013). Tox has been shown to be an additional marker for MF and SS (Zhang et al. JID 2013).

\section{AIM}

The aim of the present study was to validate this combination of blood-derived biomarkers in a validation cohort of SS, erythodermic and earlier MF, for improving diagnosis and predicting prognosis.

\section{METHODS}

Patients with a confirmed diagnosis of MF $(n=221)$ or SS $(n=156)$ and patients with other skin diseases $(n=57)$ were consecutively included between 01/2011 and 12/2015. After written informed consent, blood samples and clinical data were collected including age, sex, diagnosis with stage, LDH, CRP, CD4/CD8 ratio, counts of leucocytes, counts of lymphocytes, counts of eosinophils, Sézary cell counts, natural killer (NK) cell counts, $\mathrm{CD}^{+}$cell percentage, $\mathrm{CD}^{2} 6^{+}$cell percentage.

Peripheral blood mononuclear cells (PBMC) were isolated by density gradient (Ficoll). CD4 ${ }^{+}$T-cells were purified by using positive magnetic cell sorting. Quantification of mRNA expression profiling was established on total PBMC and CD4 ${ }^{+}$T-cells by use of SYBR Green PCR Core (Thermo Fisher Scientific Inc) with specific primer pairs (available on request). Measures of gene expression were normalized with a housekeeping gene $(\beta 2 \mathrm{M})$.

Data were analysed descriptively (means, medians and standard deviations). To compare groups, independent samples ttest and Welch's t-test were used with an alpha 0.05 (two-taled). To test the convergent and divergent validity Pearson correlation coefficients were calculated. Correlations with loss of CD7 and CD26 expression can be regarded as criterion validity, because these are the currently recommended parameters for the diagnosis of SS. To investigate the criterion validity and reliability, Reciever Operating Characteristics (ROC) curves were analyzed, areas under the curves (AUC) were calculated with thresholds of $95 \%$ specificity for each marker. Statistical analysis was performed using R statics.

\section{RESULTS}

A total of 156 patients with SS, 221 patients with MF and 57 patients with inflammatory dermatoses were included and compared. The expression of T-plastin, Twist, KIR3DL2 and Tox were statistically significant in SS and erythrodermic MF compared the other groups ( $p \leq 0.001$ and, $p \leq 0.05$, respectively). The combination of T-plastin and Twist for the diagnosis of MF or SS had $\mathbf{9 5 . 2 \%}$ specificity and $84 \%$ sensitivity. The addition of KIR3DL2 into the diagnosis model increase its sensitivity to $94 \%$ but decreased the specificity to $90 \%$. Patients expressing KIR3DL2 had significantly lower time to relapse compared to the other groups : $\sim$ prognostic marker

Patient characteristics

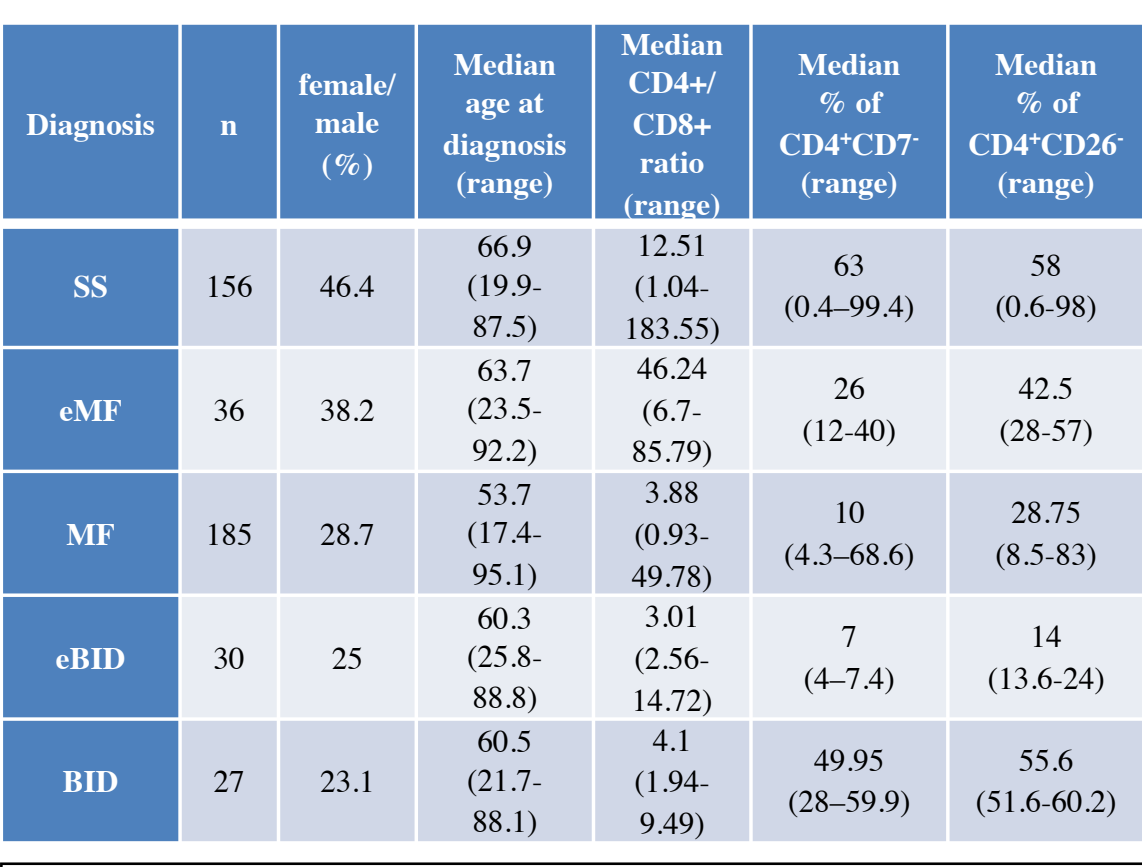

SD: standard deviation; SS: Sézary syndrome; eMF: erythrodermic mycosis fungoides; MF mycosis fungoides; eBID: erythrodermic benig inflammatory dermatosis; BID: benign inflammatory dermatosis

Correlation of the markers with gold standards

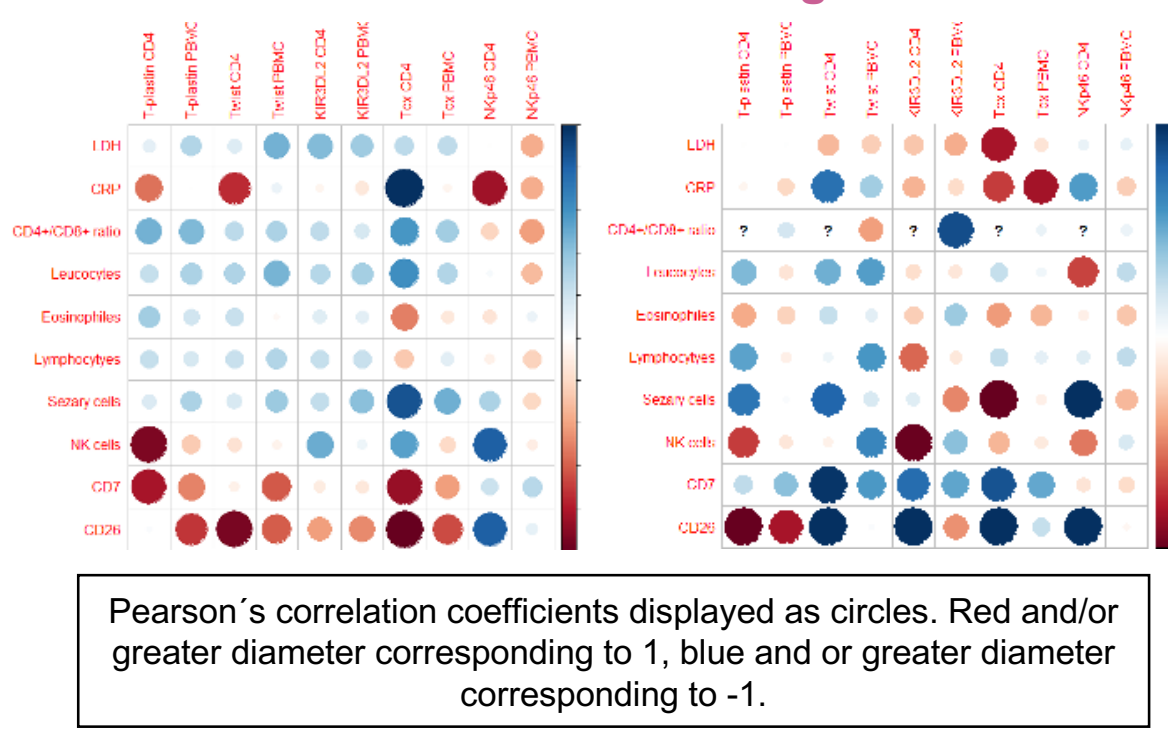

Reciever Operating Curves of the markers

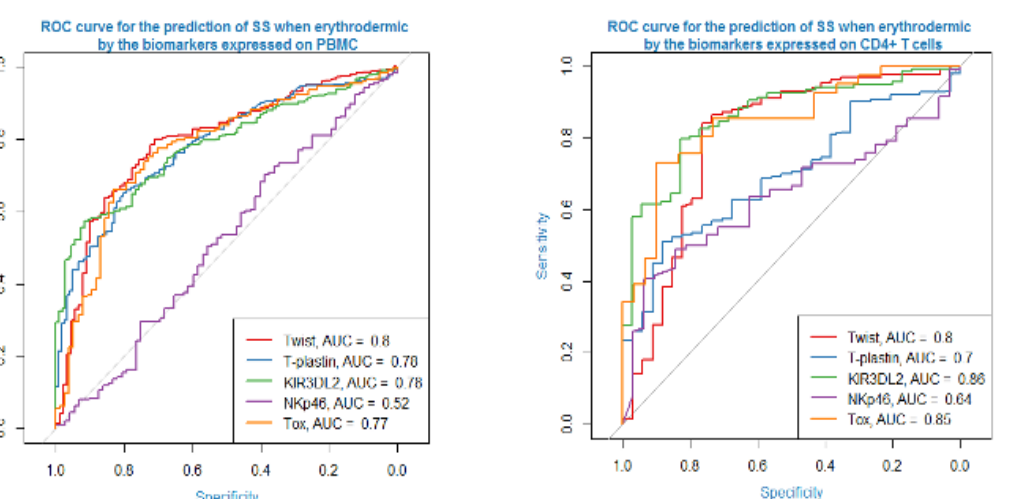

Reciever Operating Characteristics (ROC) curves displaying the ability of the markers to distinguish between erythrodermic MF and SS
*Corresponding author : laurence.michel@inserm.fr

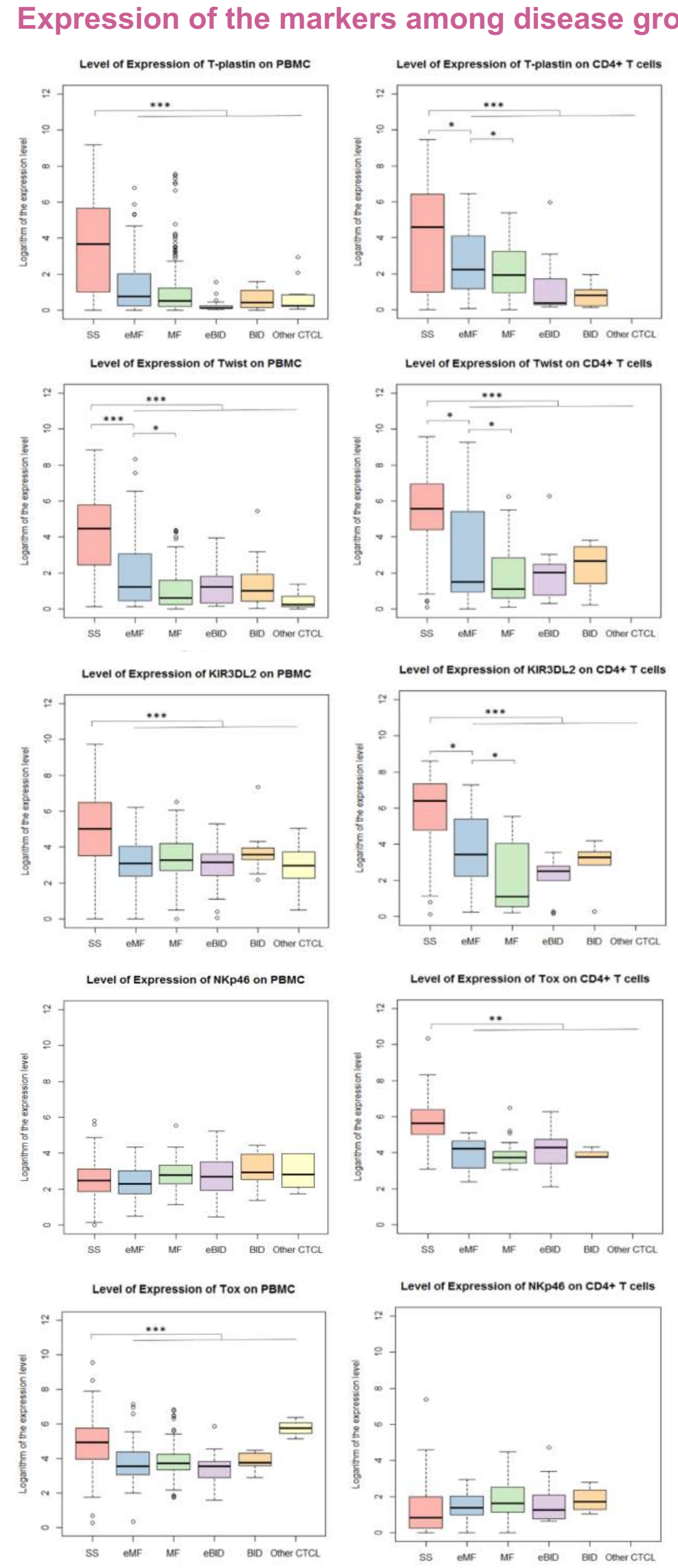

SS: Sézary syndrome; eMF: erythrodermic mycosis fungoides; MF mycosis fungoides; eBID: erythrodermic benign inflammatory ermatosis; BID: benign inflammatory dermatosis; CTCL: cutaneous T-cell-lymphoma; Welch's t-test: *: $p<0.05 ;{ }^{* *}: p<0.01 ;{ }^{* * *}: p<0.001$

Prognostic value of KIR3DL2 KIR3DL2 PBMC

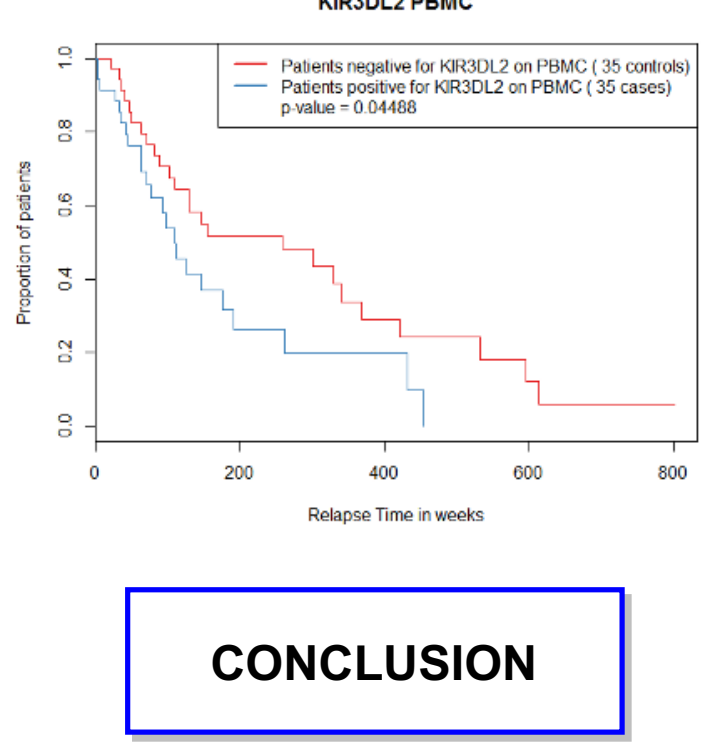
de Paris

References

Michel L et al. Use of PLS3, Twist, CD158k/KIR3DL2, and NKp46 gene expression combination for reliable Sézary syndrome diagnosis. 2013 Blood 